\title{
Femoral Periprosthetic Fracture Treatment Using the Ortho-Bridge System: A Biomechanical Study
}

\author{
wen wang ( $\nabla$ wangwenqfs@sina.com ) \\ 8986-0781 \\ Yuntao Long \\ Shandong First Medical University \& Shandong Academy Medical Sciences \\ Yubin Qi \\ The First Affiliated Hospital of Shandong First Medical University Shandong Provincial Qianfoshan Hospital \\ Guilai Zuo \\ The First Affiliated Hospital of Shandong First Medical University Shandong Provincial Qianfoshan Hospital \\ Qingjie Zhang \\ Tianjin Weiman Biomaterials Co.,Ltd \\ Zhenlin Liu \\ Tianjin Weiman Biomaterials Co.『Ltd
}

The First Affiliated Hospital of Shandong First Medical University Shandong Provincial Qianfoshan Hospital https://orcid.org/0000-0002-

\section{Research article}

Keywords: Biomechanical study, Ortho-Bridge System, Locking Compression Plate/Locking Attachment Plate, Vancouver B1 periprosthetic fracture

Posted Date: September 27th, 2021

DOl: https://doi.org/10.21203/rs.3.rs-910432/v1

License: (c) (1) This work is licensed under a Creative Commons Attribution 4.0 International License. Read Full License 


\section{Abstract}

Background: We undertook a comparative biomechanical study of type B1 fractures around the femoral prosthesis following cemented hip arthroplasty using the Ortho-Bridge System (OBS) and a locking compression plate/locking attachment plate structure (LCP+LAP), and aimed to determine the effectiveness and advantages of the OBS when treating this fracture type.

Methods: An OBS fixation model was designed based on OBS and LCP+LAP fixation characteristics. The LCP+LAP combination (Group A) and three different OBS combinations (Groups B, C, and D) were used to fix a B1 fracture model with a femoral periprosthetic fracture. Axial compression and torsion experiments were then performed using simple and comminuted fracture models. We conducted axial compression failure, model stiffness, and torsion angle tests, and tested the vertical load of final failure.

Results: When simulating simple oblique fractures, no significant difference was found in terms of stiffness between the four groups in the axial compression experiment $(P=0.257)$. The torsion angle of the LCP+LAP system was significantly higher than that of the OBS $(P<$ $0.05)$; however, there was no significant difference in the torsion angle between the OBS combinations $(P>0.05)$. Axial compression experimental data showed that stiffness in the three OBS combinations was higher than that in the LCP+LAP system $(P=0.000)$. Torsion angles of the three OBS combinations were smaller than those of the LCP+LAP system $(P<0.05)$. In the axial compression failure test, the fixed failure mode in the LCP+LAP system involved destruction of the contact cortex at the fracture site, while the failure modes in the three OBS combinations involved destruction of the contact cortex at the fracture site and the fracture around the screws above the osteotomy.

Conclusion: Compared with the LCP+LAP, the OBS showed superior biomechanical results. Furthermore, the OBS has the advantage of multiple choices and high flexibility of combinations. Stress dispersion was helpful in avoiding internal fixation failure during early postoperative functional exercise.

\section{Background}

In 1954, Holovitz and LeNobel first described periprosthetic fractures (PPFx) as involving those above, below, or around implant prostheses [1]. The number of hip replacement surgeries reported by the National Joint Registry has increased annually, and 91,833 operations were conducted in 2015 [2]. The rise in PPFx is directly related to the increasing frequency of primary joint replacement operations every year [3]. With an increase in the number of hip replacements, the number of PPFx during and after surgery is expected to increase [4-7]. According to related reports, the incidence of PPFx is $11 \%$ after the first total hip replacement and $7 \%$ after a semi-joint replacement [8-9].

The Vancouver classification proposed by Duncan [10] is the most commonly used classification for femoral PPFx that includes the anatomical site of fracture, prosthesis stability, and bone stock quality, which is helpful information when determining a final fracture treatment plan [11]. When femoral prostheses are well fixed (type A, type B1, and type C), PPFx can be achieved either non-surgically or through an open reduction and internal fixation (ORIF) procedure, whereas types B2 and B3 require prosthesis revision [4,12-13]. The most common treatment for type B1 PPFx using the Vancouver classification has been reported to be an ORIF procedure using locking plates, steel cables, or allogeneic cortical supports [13].

Although the main treatment for Vancouver B1 fractures is open reduction and internal fixation, no consensus has been reached concerning the optimal method of reduction and fixation [14-17]. The Ortho-Bridge System (OBS) is a new internal fixation system [18-19] developed by Tianjin Weiman Biomaterials Co., Ltd. It is made of titanium alloy and consists of connecting rods, locking screws, locking nuts, fixing blocks, and common screws (Fig. 1). As the basic units of the OBS, nails, rods, and blocks can be individually combined and configured and fixed using either single, double, or multiple rods. Moreover, the OBS can be locked and unlocked. The individualised combination of nails and rods makes it possible for diversified fixed modes. At the same time, it allows three-dimensional (3-D) fixation with multi-bar and steering nails, improves the pull-out strength, and creates a larger personalised application space for the treatment of particularly complex fractures. Biomechanical and clinical analysis studies have shown that internal fixation using the OBS was effective in treating long bone fractures $[18,20]$, and the OBS has been applied in fixation of upper and lower limbs or pelvic fractures. Some orthopaedic surgeons in China have attempted to use the OBS to treat femoral PPFx. Although good results have been achieved, the application of the OBS to treat femoral PPFx has been greatly limited due a scarcity of published data. Therefore, in this study, we refer to various previously designed PPFx internal fixation concepts. We attempted to meet the requirements of multi-angle fixation and ensure fixation around a prosthesis in as far as possible, and we attempted to locate the double cortical screw access around the prosthesis as often as possible [21]. Based on the advantages of the OBS, we designed a PPFx model of the OBS and conducted biomechanical analysis and comparison experiments with the model, which was made using the LCP + LAP fixation system that is in common use worldwide (Fig. 2).

This experiment aimed to compare the biomechanics of the OBS and the LCP + LAP fixation system used to treat a Vancouver B1 fracture model with femoral PPFX, and to explore the effectiveness of the OBS in treating this type of fracture and the advantages of this internal 
fixation system.

\section{Methods}

\section{Experimental materials}

The following materials were used in this study: 24 artificial standard femora (third-generation compound femora, medium 3304; Sawbones, a department of the Pacific Research Laboratory in Vashon, Washington); a 12-hole Cindy steel plate and a corresponding attached steel plate (DePuy Synthes, Solothurn, Switzerland); and the Ortho-Bridge System, comprised of a 6 mm diameter connecting rod, a fixing block, and a fixing screw (Tianjin Weiman Biomaterials Co., Ltd.).

\section{Experimental grouping}

This experiment comprised the four following groups: (i) Group A, a LCP + LAP fixed group; (ii) Group B, a bridging double-rod and doublecortex fixation group; (iii) Group C, a bridging double-rod and single-cortex fixation group; and (iv) Group D, a bridging single-bar cross-fixed group. Six experimental models were constructed for each group. The number of experimental models was determined in accordance with similar experiments undertaken internationally [21], and also met requirements for statistical analysis.

\section{Model construction}

This experiment was completed in the Newton Laboratory of Tianjin Weiman Biomaterials Co., Ltd. First, orthopaedic specialists performed a standard femoral neck osteotomy using Sawbones with a swing saw at a height of $10 \mathrm{~mm}$ near to the lesser trochanter. A hole was cut and the medullary cavity was opened using a medullary cavity file. The medullary cavity was enlarged to match the diameter of the medullary cavity expander. After placing a cement restrictor at the distal end of the medullary cavity, an appropriate amount of Heraeus bone cement was manually applied to fill the cortex, and the prepared femoral stem prosthesis was implanted. Twenty-four Sawbone femora were made. First, we used a 12-hole Cindy's plate, a corresponding attached plate, and the OBS for internal fixation. The bones were then sawed, and four groups of models were made to treat the B1 fractures. The length of the OBS connecting rod was consistent with a standard 12hole locking steel plate, which was placed at the planned position under the femoral trochanter. In the bridge double-cortex fixation group, the bicortical screw channel at the upper end of the fracture was located under fluoroscopic observation (Fig. 2).The specific fixing requirements of each group were as follows:

(i) Group A, the LCP + LAP fixation group

We selected a Synthes locking (12 holes) wide dynamic compression plate, an attachment plate, and corresponding locking screws. Four 5$\mathrm{mm}$ locking screws at the proximal end of the fracture, including two double-cortex locking screws were attached to the plate, two other screws were monocortical, and there were three 5-mm double-cortex locking screws at the distal end of the fracture.

(ii) Group B, the bridging double-rod and double-cortex fixation group

Two connecting rods (length, $22 \mathrm{~cm}$; diameter, $6 \mathrm{~mm}$ ), and three single-rod and single-hole fixation blocks and double-cortex locking screws were used to fix the proximal end of the fracture from top to bottom. Near the fracture end, a double-rod single-hole fixation block and a single-cortex locking screw were used for fixation. The distal end of the fracture was fixed with three double-rod single-hole fixation blocks and three double-cortex locking screws.

(iii) Group C, the bridging double-rod single cortex fixation group

Two connecting rods (diameter, $6 \mathrm{~mm}$ ) were selected, along with three single-rod single-hole fixation blocks, one double-rod single-hole fixation block, and four screws, which were all single cortex locking screws from top to bottom at the proximal end of the fracture. We used three double-rod single-hole fixation blocks and three double-cortex locking screws for fixation at the distal end of the fracture.

(iv) Group D, the bridging single-rod cross-fixation group

One connecting rod (length, $22 \mathrm{~cm}$; diameter, $6 \mathrm{~mm}$ ) and one connecting rod with a shorter length were selected, and the two rods were separated at a certain distance. There were four single-rod and single-hole fixed blocks and four single-cortex locking screws at the proximal end of the fracture. From top to bottom, the first and third screws were located on the long connecting rod, and the second and fourth screws were located on the short connecting rod. Spacing of these two sets of screws was staggered at approximately $90^{\circ}$, resulting in fracture, and four double-cortex locking screws were used. From top to bottom, the first and second screws were located on the short connecting rod, 
and the third and fourth screws were located on the long connecting rod. Spacing of these two sets of screws was staggered at approximately $90^{\circ}$ (Fig. 3).

\section{Biomechanical test}

Dental tray powder (polymethyl methacrylate formaldehyde) was poured on the distal femur, and an appropriate iron cup was used to fix the distal femur in the experiment. Outside the bone cement and $2.5 \mathrm{~cm}$ away from the distal end of the femoral stem prosthesis, an industrial wire saw was then used to cut a $45^{\circ}$ oblique fracture line from the upper lateral side to the lower medial side (simulated anatomical reduction and Vancouver B1 simple fracture fixation), so that the supporting effect of internal fixation could be prevented during the axial compression test [21]. All samples were subjected to axial compression and torsion tests, and the stiffness and torsion angle values of the different system groups were obtained when treating simple fractures. An industrial milling machine was then used to form a 5-mm bone gap at the sawing bone site to simulate the comminuted fracture model, and two groups of axial compression stiffness and the torsion angle data were obtained when testing and treating complex fractures again. Finally, all samples were subjected to axial compression failure, and failure compression force values were obtained. In this study, simple fracture was defined as the initial saw bone, comminuted fracture was defined as the 5-mm bone gap, and the axial compression failure experiment was defined as the continuous application of load during axial compression until irreversible failure of the implant or the femur occurred.

\section{Experimental tests}

\subsection{Axial compression test}

The experiment was performed using a microcomputer-controlled electronic universal testing machine (Equipment model E45.105, Fig. 4). The cast iron cup at the distal femur was matched and fixed to the testing machine, the femoral head prosthesis at the proximal femur was in contact with the white polyethylene cylinder of the testing machine, and the experiment was performed under an initial vertical load of $\bigotimes$ $100 \mathrm{~N}$, amaximum vertical load of $1000 \mathrm{~N}$, and a displacement loading rate of $8 \mathrm{~mm} / \mathrm{min}$, and the load-displacement curve was obtained. The slope of the curve was obtained using computer software囚TW-Elite囚connected to the testing machine.

In the crushing fracture test, changes in the stiffness of the fracture end after contact were observed, and the load was approximately $500 \mathrm{~N}$. The stiffness values without contact were compared to eliminate test-related influencing factors. The load level was lower than the physiological level in many daily life activities; however, to prevent permanent damage to the sample during the stiffness test, a lower load level was selected [21].

\subsection{Torsion test}

This experiment was performed using a torsion testing machine (Equipment model ND-200; Fig. 6). The femur was horizontally installed on the equipment, the cast iron cup at the distal end of the femur was matched and fixed to the testing machine, and the femoral head was fixed with clamps at both ends of the testing machine. The experimental conditions comprised a maximum of $10 \mathrm{~N} \cdot \mathrm{m}$ and a loading rate of $90^{\circ} / \mathrm{min}$. P-main computer software was connected to the testing machine, which was used to obtain the torque-rotation curve, and a lower load was selected to avoid permanent damage to the sample during testing.

\subsection{Axial compression failure test}

The axial compression failure test was the final failure test, which was conducted using a microcomputer-controlled electronic universal testing machine (equipment model: E45.105). The cast iron cup at the distal femur was matched and fixed to the testing machine, the femoral head prosthesis at the proximal femur was in contact with the white polyethylene cylinder of the testing machine, and the vertical initial load was applied to the femoral head within $100 \mathrm{~N}$, and the displacement loading rate was $8 \mathrm{~mm} / \mathrm{min}$, until the implant or femur was irreversibly damaged, and the failure mode and verticality during failure were recorded.

\section{Experimental evaluation}

The stiffness value indicates the capacity of an implant to resist deformation in the elastic stage, and it is used as a standard evaluation of axial compression. Under the same axial pressure, the greater the stiffness value, the smaller the deformation of the plant and the firmer it is. The torsion experiment was used to compare the difference in torsion angle between different implanted structures under the same torque load, that is, torsion stiffness. Under the same torsion force, the torsion angle of the endophyte decreases, which means that the endophyte is stronger. The aim of the axial compression failure test was to detect the maximum load that the structure of the inner plants could bear. The greater the maximum load, the stronger the resistance of the inner plants to destructive force and the better the overall strength. 


\section{Statistical analysis}

To analyse the differences among all of the structures, after ensuring the normal distribution of the test data, the data were analysed using one-way ANOVA, and the significance level was set at $\mathrm{P}<0.05$. When the variance between the control groups maintained homogeneity, the Bonferroni method was used for multiple comparisons in pairs, while the Tamhane method was used for multiple comparisons when the variance between the control groups did not maintain homogeneity. When analysing the comparison between the simulated simple fracture group and the simulated comminuted fracture group, a t-test was performed, and the Bonferroni significance level was adjusted to $\mathrm{P}<$ 0.0125. The adjustment value was calculated through dividing the P-value of the $95 \%$ confidence interval by the number of constructions compared, that is, Bonferroni $=$ the P-value of the $95 \%$ confidence interval/number of constructions $=0.05 / 4=0.0125$.

\section{Results}

\section{Axial stiffness}

In the simulation of the simple fracture, the axial stiffness of all structures ranged from 655.9 to $1113.5 \mathrm{~N} / \mathrm{mm}$. In the simulation of the comminuted fracture, the axial stiffness ranged from 75.5 to $176.8 \mathrm{~N} / \mathrm{mm}$ (Fig. 5). In the simulation of the simple fracture, there was no statistically significant difference in the axial stiffness data for each structure $(P=0.257)$. The axial stiffness of the three OBS groups was higher than that of the LCP + LAP group $(P=0.000)$. The axial stiffness of the bridging single-rod cross fixation group was higher than that of the bridging two-rod and double-cortex fixation groups $(P=0.02)$. There were no significant differences in the data concerning the other groups $(P>0.05)$. When comparing the simulated simple fracture group and the simulated comminuted fracture group, the axial stiffness of each structure in the simple fracture group was higher than that in the comminuted fracture group $(P<0.0125)$.

\section{Torsion angle}

In the simulation of the simple fracture, the torsion angle of all structures ranged from $4.53^{\circ}$ to $7.88^{\circ}$, and in the simulation of the comminuted fracture, the torsion angle of all structures ranged from $4.8^{\circ}$ to $9.18^{\circ}$ (Fig. 6 ). Data analysis during the simulation of simple fractures showed that the torsion angle of the LCP + LAP system was significantly higher than that of the three OBS combinations $(P<$ $0.05)$. Bridge data differences between the three combination types were not statistically significant $(P>0.05)$. In the comminuted fracture simulation data, the LCP + LAP system torsion angle above the bridge double-rod double-cortex $(P=0.024)$ and the bridge double-rod torsion angle of the single-cortex combination were clearly higher than that of the bridging double-rod double-cortex combination $(P<0.05)$. There was no statistically significant difference within the data set, and there was no statistical significance in the comparison between the simulated simple fracture group and the simulated comminuted fracture group $(P>0.0125)$.

\section{Axial failure test load}

In the final failure test, the axial failure load of the sample ranged from $3756.29 \mathrm{~N}$ to $5124.34 \mathrm{~N}$ (Fig. 7). The failure pressure of the LCP + LAP system was lower than that of the bridged two-rod double-cortex and bridged two-rod single-cortex combinations $(P<0.05)$. The failure pressure of the bridged two-rod double-cortex combination was higher than that of the bridged two-rod single-cortex and single-rod crossover fixation combination $(P<0.05)$, whereas the failure pressure of the bridged two-rod single-cortex combination was higher than that of the bridged single-rod crossover fixation combination $(P<0.05)$.

In the axial compression failure test, with an increase in the vertical load, the fracture gap decreased to facilitate contact between the two; the steel plate and bridge were fixed in a progressive bending position, and irreversible failure subsequently occurred. After all samples were damaged, several failure modes seen in all samples and groups were obtained, although fracture of the permanent steel plate, attached steel plate, and connecting rod was not observed. Moreover, no screw was loose, pulled out, or damaged, and the bone cement femoral stem was loose.

The fracture modes in all groups were similar (Fig. 8), and the failure modes in the LCP + LAP group were similar, that is, there was destruction of the upper cortex at the contact point of the medial femoral space, with no fracture of the femur was found. The failure mode of the OBS in the double-rod and double-cortex group showed not only destruction of the upper cortex at the contact point of the medial bone gap, but also a stable transverse crack near the first bicortical screw on the lateral femur and a longitudinal fracture crack between the first bicortical screw and the second screw on the medial femur (3 of 6 samples). The failure mode of the OBS with double rods and a single cortex was similar to that of the combination of double rods and double cortices. Both had cortical failure of the LCP system, which originated from an oblique fracture (from top to bottom) from the first to the third screw in the proximal femur (6 samples) and from a stable transverse crack near the third screw (3 of 6 samples). Cortical damage to the LCP system and the stable oblique crack of the second screw originating from the long rod also occurred in the single-rod cross-fixation group of the OBS. 


\section{Discussion}

Treatment of femoral PPFx is a major challenge in modern orthopaedics and adequate fixation around the prosthesis and the choice of effective internal fixation remains clinically demanding.

This study compared the biomechanics of the LCP + LAP and the OBS for fixation of femoral PPFx. First, a load-displacement curve was obtained through an axial compression test, with the slope of the stress-displacement curve considered to demonstrate stiffness of the internal fixation model as a whole (the fracture end contracts under load). The initial axial stiffness of the LCP + LAP fixation group was not significantly different from that of the group in Lenz et al.'s study [25]. The stiffness measurements and torsion angles in our study can be used as biomechanical parameters, especially for the OBS. When simulating simple fractures in this experiment, no apparent difference in stiffness was observed among the four groups in the axial compression experiment $(P>0.05)$; however, the torsion angle of the LCP + LAP fixation group was higher than that of the OBS group $(P<0.05)$, which indicated that the biomechanics of the three OBS combinations were better. When simulating the comminuted fracture, the structural stiffness of the three OBS combination groups was higher than that of the LCP + LAP group $(P<0.05)$, and the torsion angle of the LCP + LAP group was also higher than that of the bridging double-rod and doublecortex group $(P<0.05)$, indicating that the biomechanics of the OBS combination groups were still better than those of the LCP + LAP fixation group. However, when crushing a fracture, the axial compression stiffness was significantly lower than that of a simple fracture ( $P<$ 0.0125). Lenz et al. [22] reported that because the support of the cortex at the fracture was reduced, an implant load was needed at this time; therefore, internal fixation was very important for axial stability, especially for crushing a fracture. Finally, the results of the axial compression failure test showed that the OBS had more fractures on the surface, but its failure mode indicated that stress could be dispersed to the femur when the OBS was used in the femur during axial compression; therefore, this fracture failure mode became evident, while it could be inferred that the failure mode of the locking steel plate was concentrated on the steel plate, which led to the steel plate bearing more stress than the femur. This is consistent with the stress distribution assessment of the finite element analysis of these two systems in the femur by Xiong Ying [18].

Kammerlander et al. [23] showed that patients aged $\geq 75$ years who received treatment for hip fractures were unable to maintain postoperative weight-bearing limits, with $69 \%$ of patients exceeding more than twice the prescribed partial weight-bearing limits; therefore, the goal of treating periprosthetic fractures must be to facilitate immediate full weight-bearing. During gait, an internal fixation system needs to carry 2 to 2.4 times the weight of an individual [24-25]. The destructive test results in this study were $>3000 \mathrm{~N}$, which was sustainable, but the stress concentration of the steel plate can easily cause fatigue during early rehabilitation exercises, leading to fracture of the internal fixation. The effective stress dispersion in the OBS system is more suitable for early functional exercise.

Challenges when treating PPFx after cemented hip arthrosis concern fixation around the prosthesis. Due to the inconvenience of doublecortex screw placement and the relative imbalance between proximal and distal fixation, pull-out of single cortex screws may occur in internal fixation. Fulkerson et al. [26] observed early failure and axial displacement of the single cortex locking screw under cyclic loading, and other biomechanical studies have shown that double-cortex screw internal fixation has advantages over single cortex screw or cortical ligation alone in proximal fixation of PPFx [22, 27-31]. In this study, double-cortex fixation of the OBS was guided using a C-arm machine. Moreover, because the orientation and the corresponding angle of the OBS connecting block could be adjusted to achieve flexible screw placement, double-cortex fixation of the OBS could be undertaken around the prosthesis without using additional devices. In the OBS bridging single-cortex fixation group, proximal fractures were fixed with single-cortex screws, but no proximal screw pull-out was observed during the fracture test, and the biomechanics were similar to that of the LCP + LAP fixation group. Using an OBS flexible connecting rod in the design for the bridge single-rod cross-fixation group, two fixed-rod screws can be placed at $90^{\circ}$ in spatial distribution to better achieve stability of the fixed-rod screw, but proximal fractures still occurred with single cortex fixation, although with no screw pull-out damage during the experiment. In the axial compression experiments, the LCP + LAP fixation group stiffness did not show any difference; however, the anti-torsion performance was better. Insertion of the double-cortex screw was more resistant to pull-out under cyclic loading, which is conducive to functional exercise in early rehabilitation and avoids screw pull-out failure.

It has been reported that minimally invasive percutaneous plate osteosynthesis combined with LCP can be used as a surgical method for the treatment of Vancouver type B1 PPFx, with intraoperative advantages (shorter operation time and less blood loss) [32], and that the OBS can also be combined with Mippo technology for fracture treatment. Liangqi et al. [21] reported that the OBS combined with Mippo technology to treat ipsilateral proximal femoral and diaphyseal fractures had a high possibility of fracture healing and good functional effect. The OBS can also provide personalised internal fixation combined with 3-D printing applications for complex and challenging fractures around the prosthesis, with preoperative preparation having obvious advantages. Moreover, the OBS has many choices of fixed combinations, allowing greater flexibility for the operating surgeon who can then obtain the best implant position, especially around the prosthetic implants. 
This study had several limitations. A standard artificial femur was used in this study, which could simulate good bone reserve. Models for osteoporosis are available; however, this study did not simulate osteoporosis and low-quality bone. Patients with fractures around a femoral prosthesis comprised older adults and these patients commonly have osteoporosis. Furthermore, in vitro simulation experiments, the influence of soft tissue involvement, the short- and long-term role of soft tissue in fracture stabilization and healing, and the convenience of OBS placement and screw placement could not be evaluated. The effects of cyclic loading in vivo were also not tested in this study.

\section{Conclusion}

This study showed that the axial stiffness of the three OBS combinations did not significantly differ from that of the LCP + LAP system in simple fractures around the femoral prosthesis; however, torsion resistance was higher in the three OBS combinations than that in the LCP + LAP system. In the simulation of comminuted fracture, the axial stiffness of the OBS was better than that of the plate system, and the torsion resistance of the bridging double-rod and double-cortex system was outstanding. The failure mode of the failure experiment showed that the stress of the OBS was more dispersed, which was consistent with the findings of other studies. The overall biomechanics of the OBS combinations was good, and the diversity and flexibility of the OBS combination provided more treatment options. The bridged internal fixation system can be helpful for the clinical treatment of femoral PPFx.

\section{Abbreviations}

LCP + LAP, locking compression plate/locking attachment plate structure; OBS, Ortho-Bridge system; ORIF, open reduction and internal fixation; PPFx, periprosthetic fracture

\section{Declarations}

\section{Ethics approval and consent to participate: Not applicable}

Consent for publication: Not applicable.

Availability of data and materials: The data that support the findings of this study are available from the corresponding author upon reasonable request.

Competing interests: The authors declare that they have no competing interests.

Funding: LCP + LAP components, artificial femur, and OBS components were provided by Tianjin Weiman Biomaterials Co., Ltd. No other funding support was received for this study.

Authors' contributions: YL, writing the paper, data analysis and collection; YQ, searching the related articles and data analysis; GZ, QZ, and ZL, experimental design and operation; WW, data analysis, article revision. All authors have contributed significantly, are in agreement with the content of the manuscript, and have approved the manuscript for submission

Acknowledgements: We thank all participants in this study for their enthusiasm, tireless work, and sustained support.

\section{References}

1. Horowitz IB, Lenobel MI. Artificial hip prothesis in acute and nonunion fractures of femoral neck. JAMA. 1954;155:564-7.

2. 13th Annual NJR Report Available. at http://www.njrcentre.org.uk/njrcentre/Portals/0/Documents/England/Reports/13th\%20Annualeport/07950\%20NJR\%20Annual\%20Rep.

3. Della Rocca GJ, Leung KS, Pape HC. Periprosthetic fractures: epidemiology and future projections. J Orthop Trauma. 2011;25:66-70.

4. Berry DJ. Epidemiology: hip and knee. Orthop Clin North Am. 1999;30(2):183-90. 10.1016/s0030-5898(05)70073-0. doi.

5. Garbuz DS, Masri BA, Duncan CP. Periprosthetic fractures of the femur: principles of prevention and management. Instr Course Lect. 1998;47:237-42.

6. Lewallen DG, Berry DJ. Periprosthetic fracture of the femur after total hip arthroplasty: treatment and results to date. Instr Course Lect. 1998;47:243-9.

7. Younger AS, Dunwoody J, Duncan CP. Periprosthetic hip and knee fractures: the scope of the problem. Instr Course Lect. 1998;47:2516. 
8. Sidler-Maier C, Waddell JP. Incidence and predisposing factors of periprosthetic proximal femoral fractures: A literature review. Int Orthop. 2015;39:1673-82.

9. Meek RM, Norwood T, Smith R, Brenkel IJ, Howie CR. The risk of peri-prosthetic fracture after primary and revision total hip and knee replacement. J Bone Joint Surg Br. 2011;93:96-101.

10. Duncan CP, Masri BA. (1995) Fracture of the femur after hip replacement. Instr Course Lect. 1995;44:293-304.

11. Rayan F, Dodd M, Haddad FS. European validation of the Vancouver classification of periprosthetic proximal femoral fractures. J Bone Joint Surg. 2008;90-B:1576.

12. Parvizi J. V egari DN (2011) Periprosthetic proximal femur fractures: current concepts. J Orthop Trauma. 2011;25(Suppl 1):S77-81. doi:10.1097/BOT.0b013e31821b8c3b.

13. Pike J, Davidson D, Garbuz D, et al. Principles of treatment for periprosthetic femoral shaft fractures around well-fixed total hip arthroplasty. J Am Acad Orthop Surg. 2009;17:677-88.

14. Lewallen DG, Berry DJ. Periprosthetic fracture of the femur after total hip arthroplasty: treatment and results to date. Instr Course Lect. 1998;47:243-9.

15. Beals RK, Tower SS. Periprosthetic fractures of the femur. An analysis of 93 fractures. Clin Orthop Relat Res. 1996;327:238-46.

16. Masri BA, Meek RM, Duncan CP. Periprosthetic fractures evaluation and treatment. Clin Orthop Relat Res. 2004;420:80-95.

17. Perren SM. Evolution of the internal fixation of long bone fractures. The scientific basis of biological internal fixation: choosing a new balance between stability and biology. J Bone Jt Surg Br. 2002;84:1093-110.

18. Wang DX, Xiong $Y$, Deng $H$, et al. Biomechanical analysis and clinical effects of bridge combined fixation system for femoral fractures. Proc Inst Mech Eng H. 2014;228:899-907. doi:10.1177/0954411914548866.

19. Schwandt CS. User manual for the 4.5/5.5 mm VetFix: development of a new veterinary internal fixation system, from the prototype to the final version. Thesis, University of Bern, Switzerland, 2001.

20. Kang L, Liu H, Ding Z, Ding Y, Hu W, Wu J. Ipsilateral proximal and shaft femoral fractures treated with bridge-link type combined fixation system. J Orthop Surg Res. 2020;15:399. Published 2020 Sep 10. doi:10.1186/s13018-020-01929-7.

21. Zdero R, Walker R, Waddell JP, Schemitsch EH. Biomechanical evaluation of periprosthetic femoral fracture fixation. J Bone Joint Surg Am. 2008;90:1068-77. doi:10.2106/JBJS.F.01561.

22. Lenz M, Windolf $M$, Mückley $T$, et al. The locking attachment plate for proximal fixation of periprosthetic femur fractures-a biomechanical comparison of two techniques. Int Orthop. 2012;36:1915-21. doi:10.1007/s00264-012-1574-x.

23. Kammerlander C, Pfeufer D, Lisitano LA, Mehaffey S, Bocker W, Neuerburg C. Inability of older adult patients with hip fracture to maintain postoperative weightbearing restrictions. J Bone Joint Surg Am. 2018;100:936-41.

24. Bergmann G, Deuretzbacher G, Heller M, Graichen F, Rohlmann A, Strauss J, et al. Hip contact forces and gait patterns from routine activities. J Biomech. 2001;34:859-71.

25. Taylor SJ, Walker PS, Perry JS, Cannon SR, Woledge R. The forces in the distal femur and the knee during walking and other activities measured by telemetry. J Arthroplasty. 1998;13:428-37.

26. Fulkerson E, Koval K, Preston CF, lesaka K, Kummer FJ, Egol KA. Fixation of periprosthetic femoral shaft fractures associated with cemented femoral stems: a biomechanical comparison of locked plating and conventional cable plates. J Orthop Trauma. 2006;20:8993. doi:10.1097/01.bot.0000199119.38359.96.

27. Lenz M, Lehmann W, Wahnert D. Periprosthetic fracture fixation in osteoporotic bone. Injury. 2016;47:44-50.

28. Lenz M, Perren SM, Gueorguiev B, Hontzsch D, Windolf M. Mechanical behavior of fixation components for periprosthetic fracture surgery. Clin Biomech. 2013;28:988-93.

29. Lenz M, Perren SM, Gueorguiev B, Richards RG, Hofmann GO, Fernandez dell'Oca A, et al. A biomechanical study on proximal plate fixation techniques in periprosthetic femur fractures. Injury. 2014;45(Suppl 1):71-5.

30. Gwinner C, Mardian S, Droge T, Schulze M, Raschke MJ, Stange R. Bicortical screw fixation provides superior biomechanical stability but devastating failure modes in periprosthetic femur fracture care using locking plates. Int Orthop. 2015;39:1749-55.

31. Lenz M, Gueorguiev B, Joseph S, van der Pol B, Richards RG, Windolf M, et al. Angulated locking plate in periprosthetic proximal femur fractures: biomechanical testing of a new prototype plate. Arch Orthop Trauma Surg. 2012;132:1437-44.

32. Min BW, Cho CH, Son ES, Lee KJ, Lee SW, Min KK. Minimally invasive plate osteosynthesis with locking compression plate in patients with Vancouver type B1 periprosthetic femoral fractures. Injury. 2018;49:1336-40. doi:10.1016/j.injury.2018.05.020.

\section{Figures}

Page 8/16 


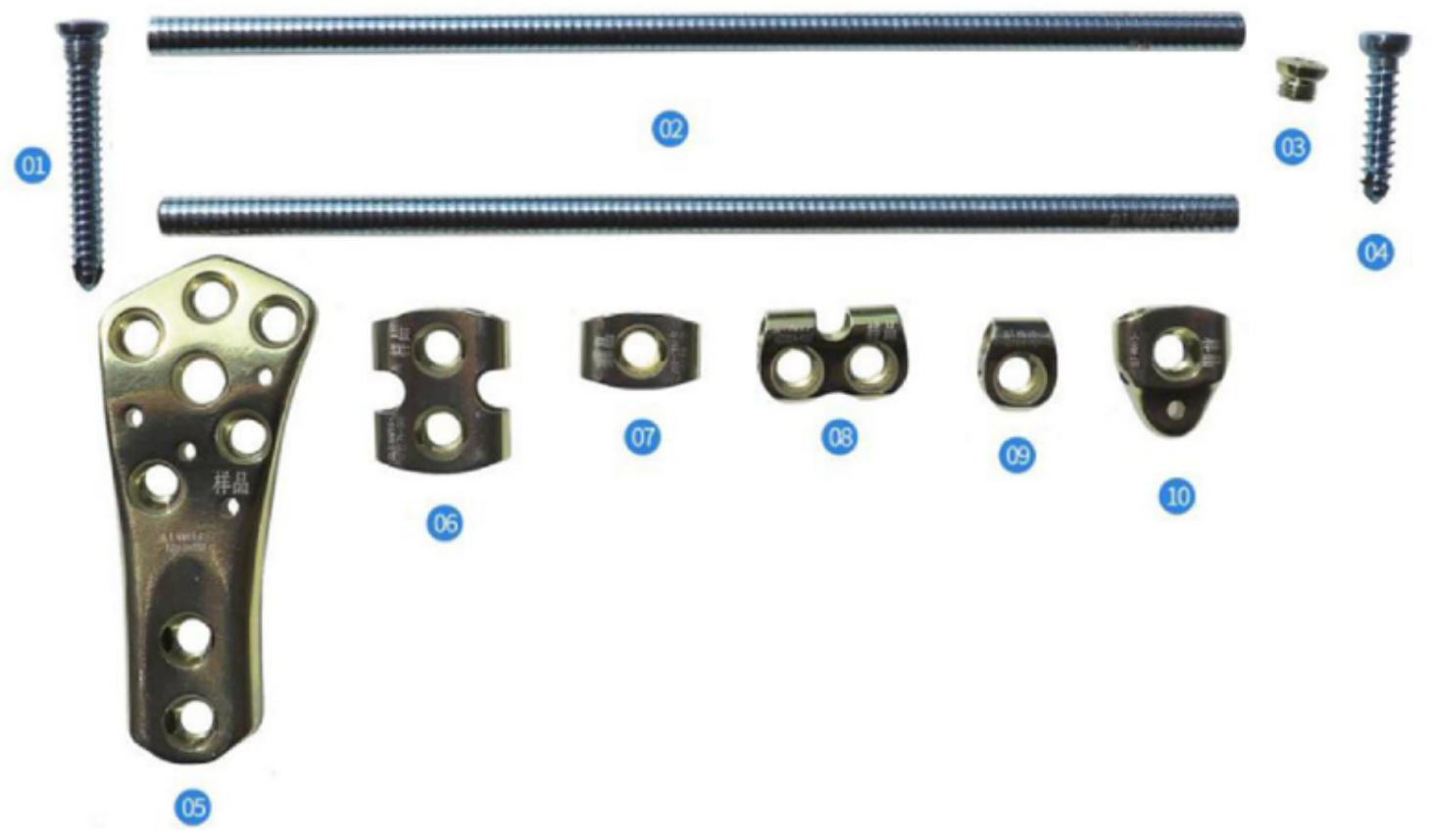

Figure 1

Figure 1

Basic unit components of the OBS (01) Locking screws, (02) connecting rod, (03) locking nut, (04) ordinary screws, (05) distal shaped piece of the femur, (06) double-rod and double-hole fixing block, (07) double-rod single-hole fixing block, (08) single-rod double-hole fixing block, (09) single-rod and single-hole fixing block, and (10) end block fixing block. 


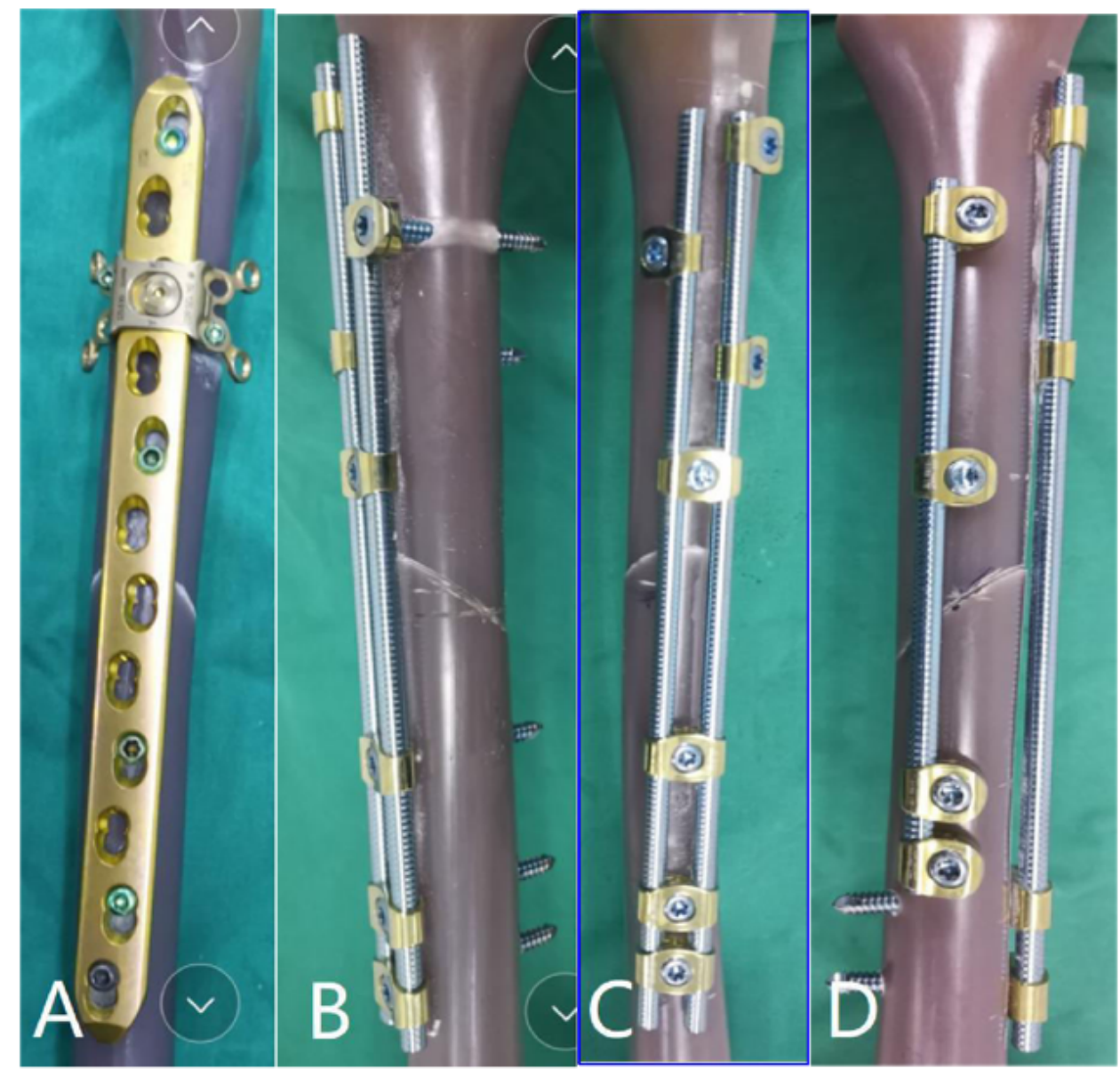

Figure 2

\section{Figure 2}

Steel plate and bridge fixing model structure Group A, LCP + LAP fixing group; Group B, bridging double-rod and double-cortex fixation group; Group C, bridging double-rod and single-cortex fixation group; Group D, bridging single-bar cross-fixation group 

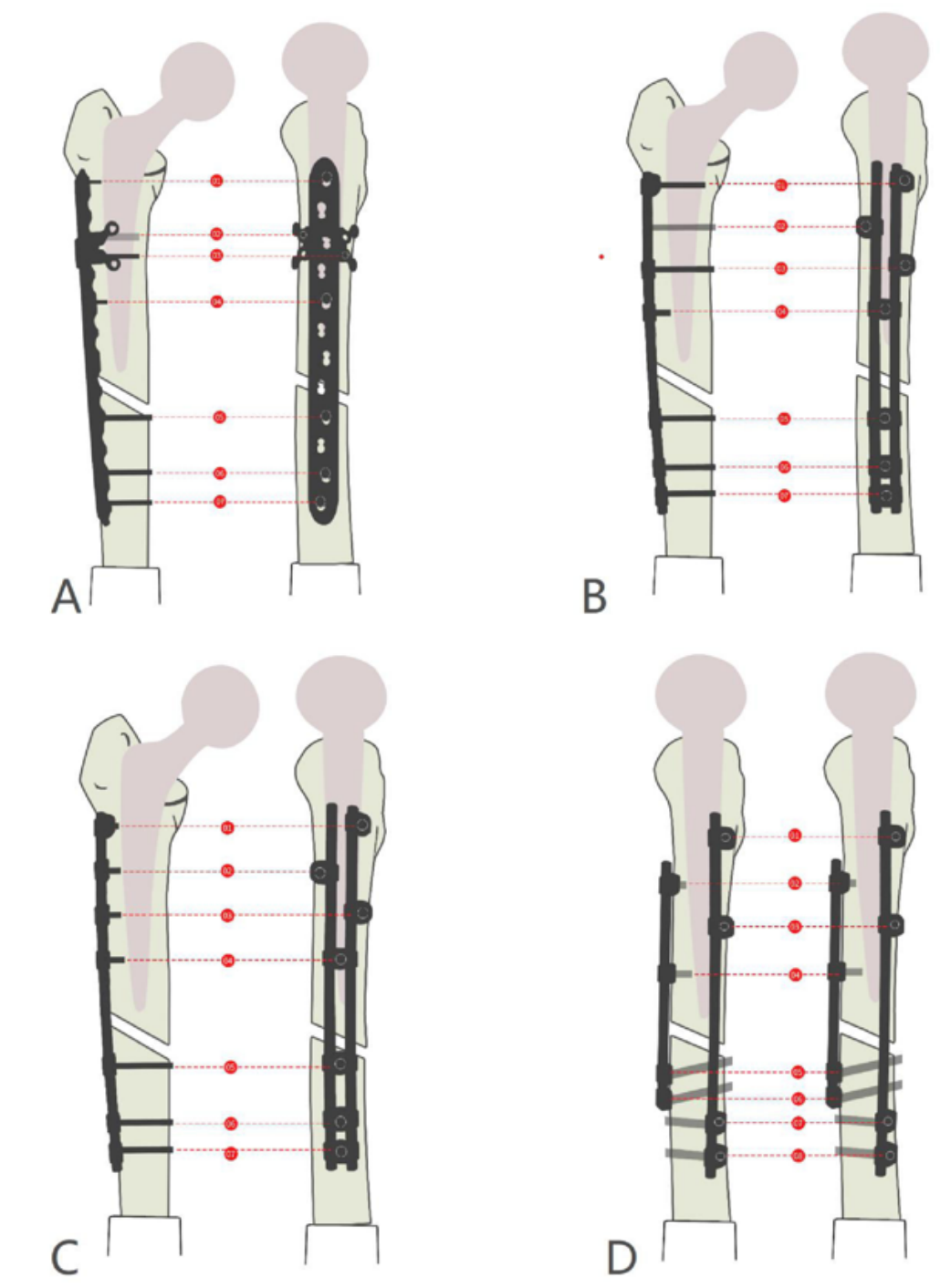

Figure 3

Figure 3

A computer-aided drawing of concrete internal fixation for Groups A-D of the experimental models 


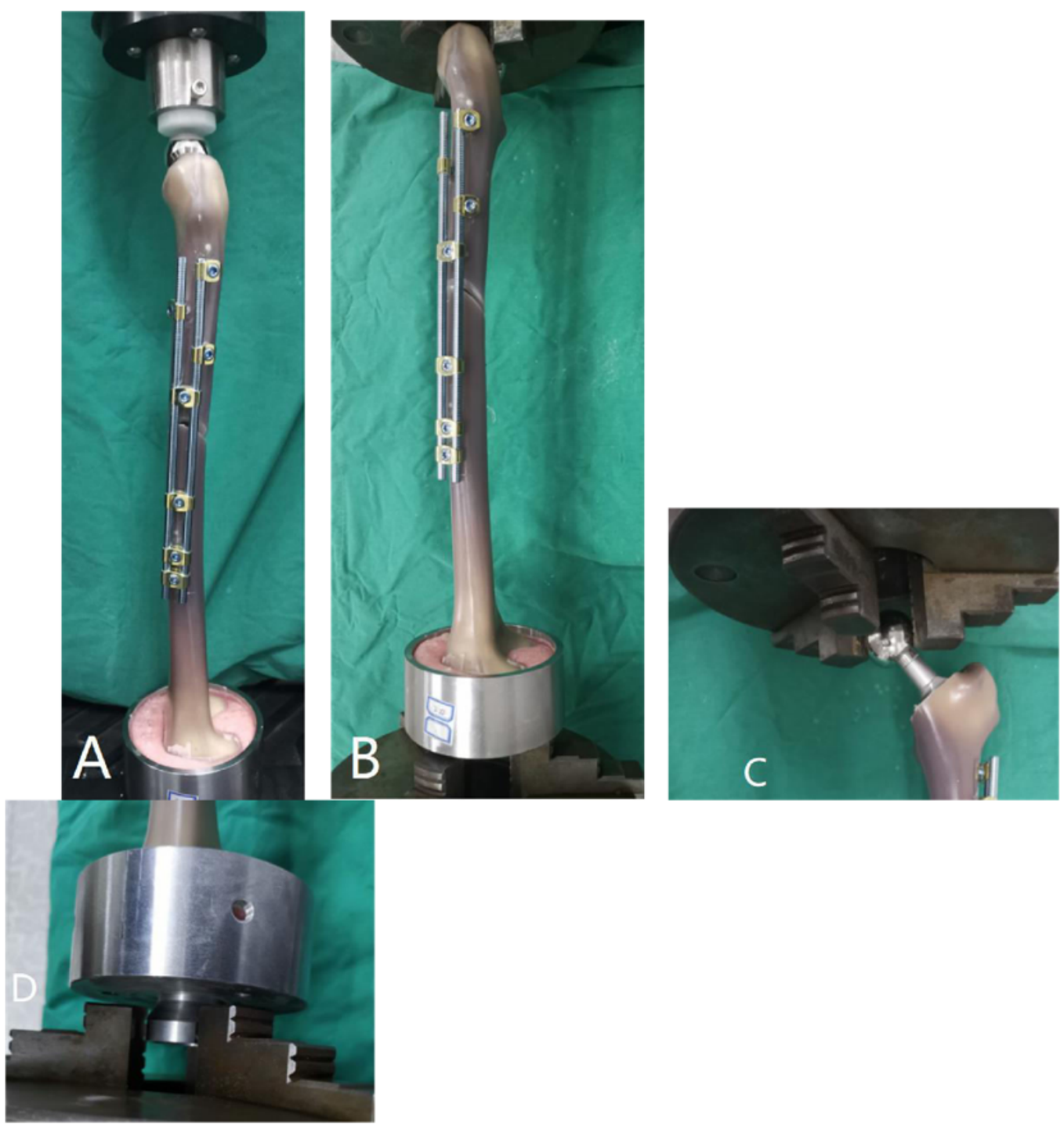

Figure 4

\section{Figure 4}

The construction model of OBS on the testing machine (A) A structural model of the OBS was tested using a microcomputer-controlled electronic universal testing machine, with a cast iron cup at the distal femur matched and fixed to the testing machine, and with the femoral head prosthesis at the proximal femur in contact with the white polyethylene cylinder of the testing machine. (B) The structural model of the OBS was tested on the torsion testing machine. (C) The proximal femoral head was fixed with clamps at both ends of the testing machine. (D) The cast iron cup at the distal femur was matched and fixed to the testing machine. 


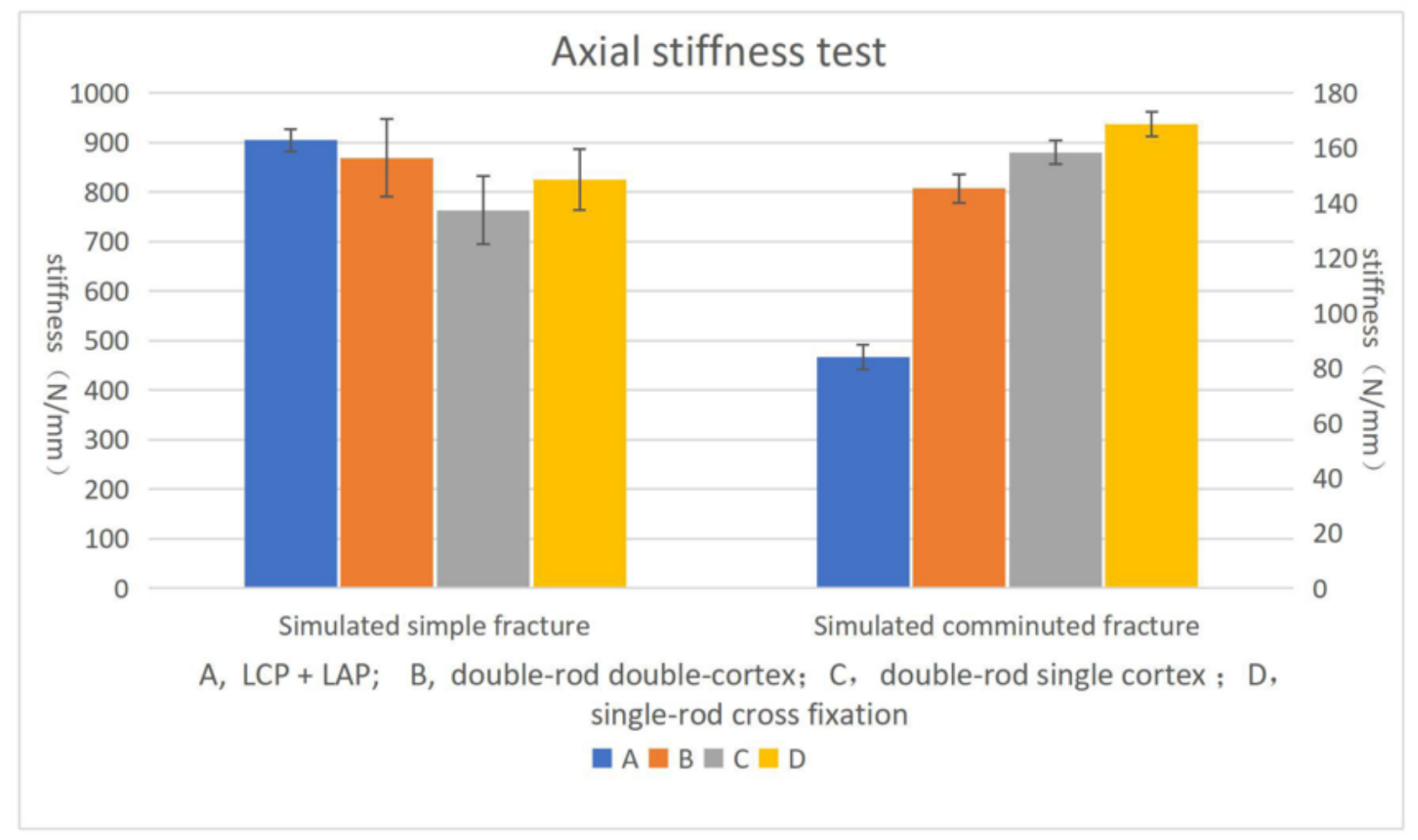

Figure 5

\section{Figure 5}

Stiffness results in the axial compression experiment The error line indicates the standard error of the average value. 


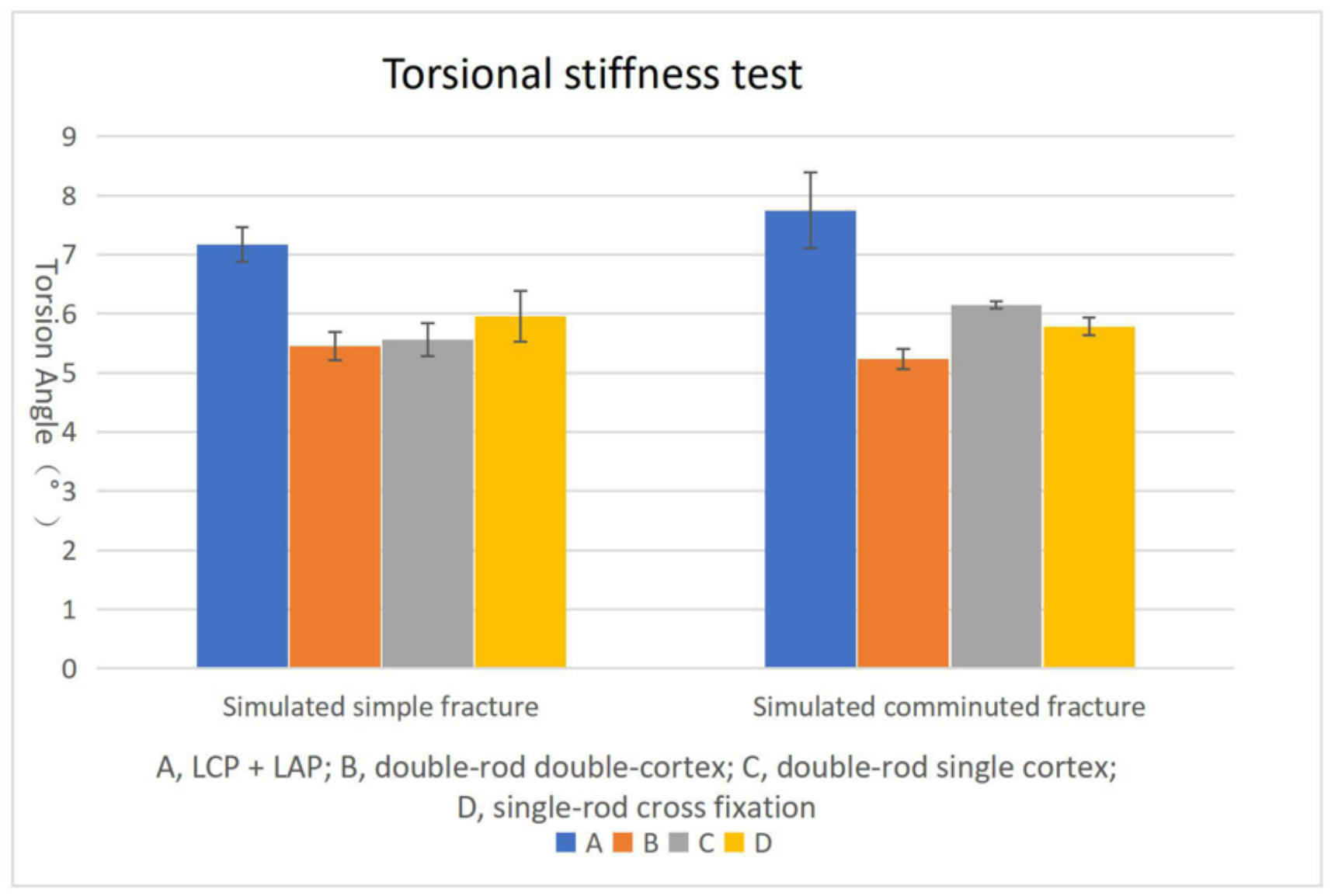

Figure 6

\section{Figure 6}

Torsion angle experiment results The error line indicates the standard error of the average value. 


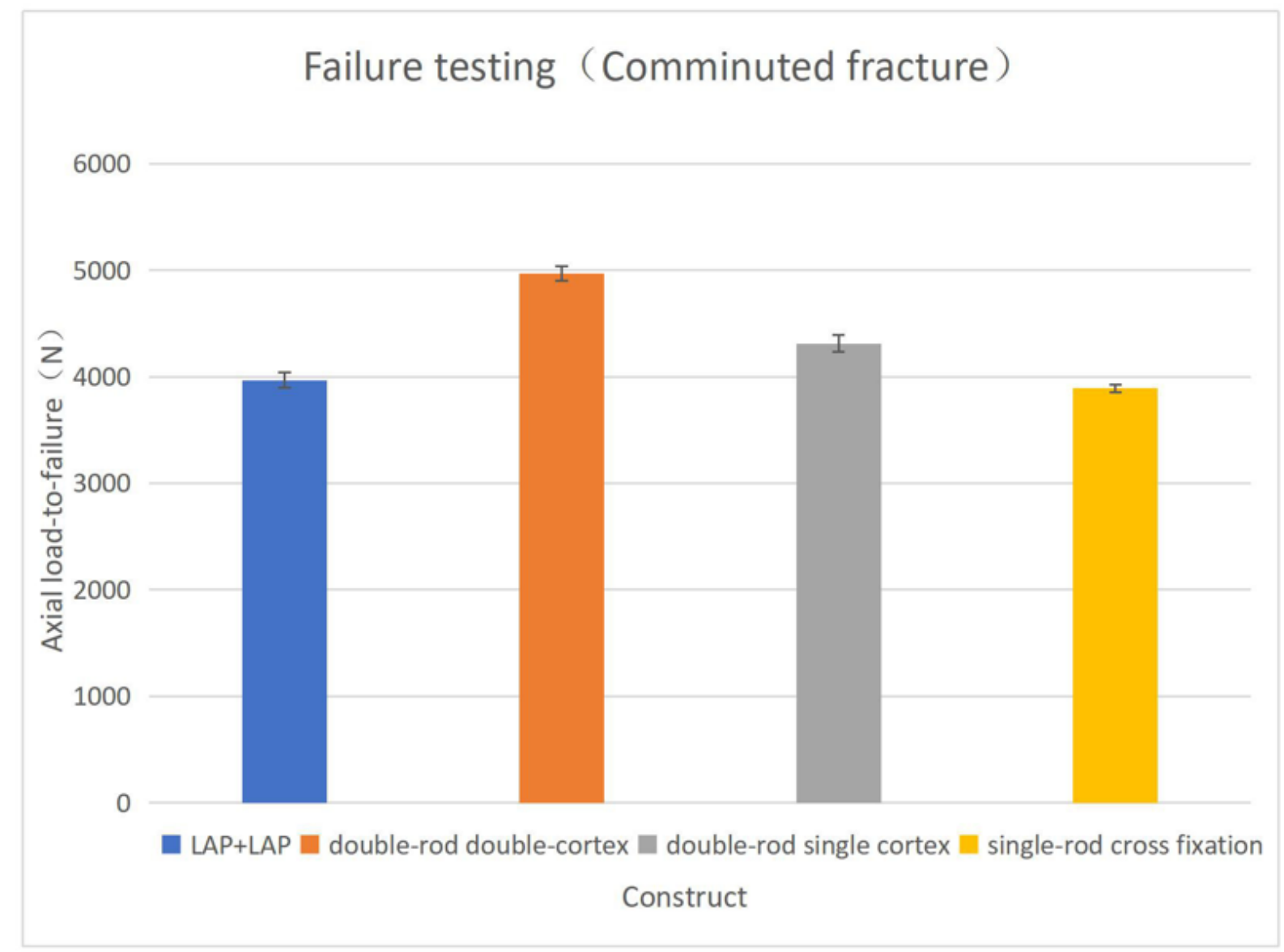

Figure 7

\section{Figure 7}

Test pressure values in the axial compression failure experiment The error line indicates the standard error of the average value. 

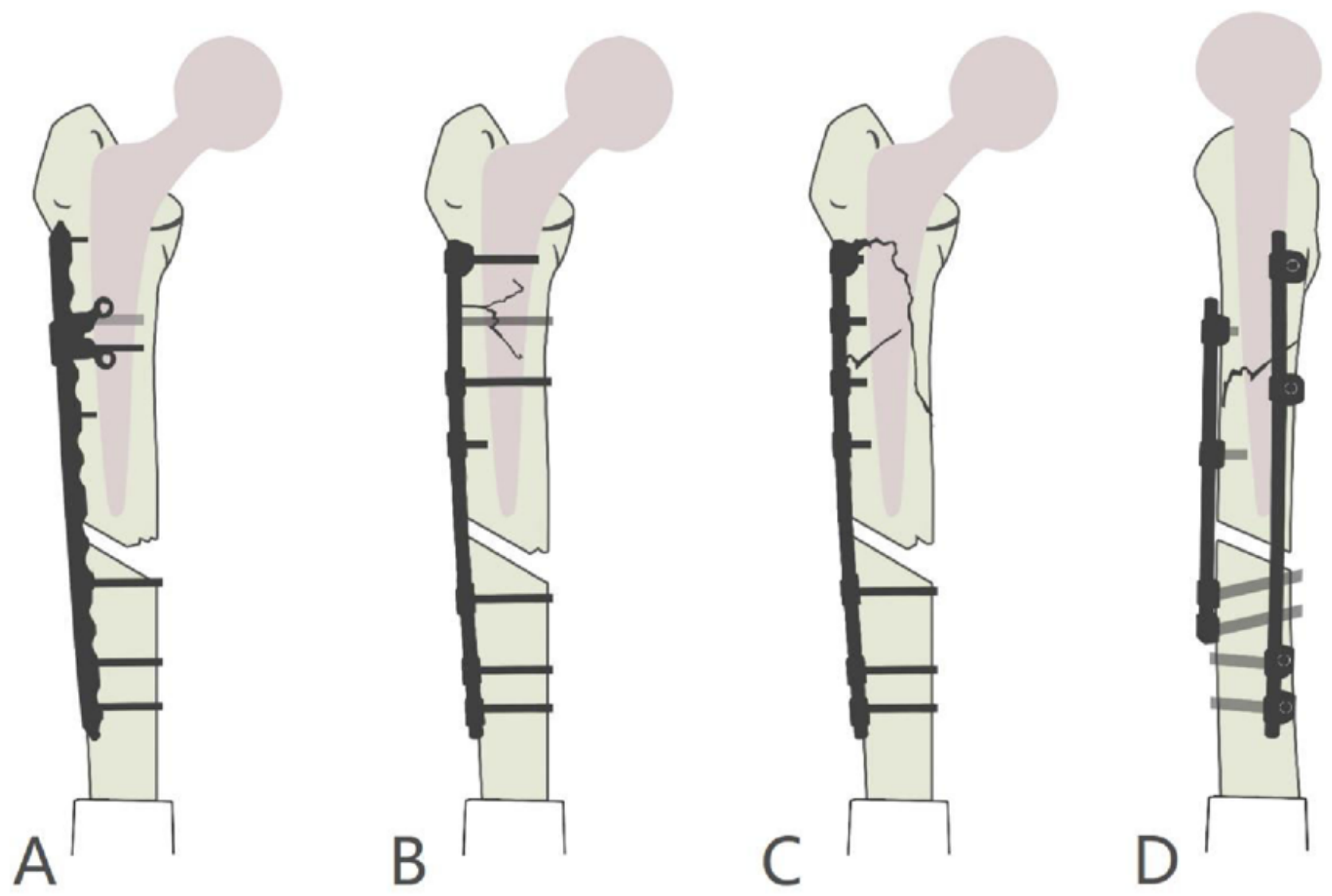

Figure 8

\section{Figure 8}

(A) The failure mode of the LCP + LAP group, (B) the failure mode of the bridged two-rod double-cortex group, (C) the failure mode of the bridged two-rod single-cortex group, and (D) the failure mode of the bridge single-bar cross-fixed group 